\title{
Mapping in Cartography
}

\author{
Miljenko Lapaine \\ University of Zagreb, Faculty of Geodesy, Kačićeva 26, 10000 Zagreb, Croatia,mlapaine@geof.hr
}

\begin{abstract}
:
The main goals of this paper are to make readers aware that the term mapping can have many different meanings and to find out the most appropriate definition of mapping in the cartographic context. Moreover, definitions of a map and cartography are derived as natural consequences.
\end{abstract}

Keywords: Mapping, Map, Cartography

\section{Introduction}

When searching the Web, which we do almost every day, we usually get the most frequent meaning of the searched term. For example, according to Wikipedia (2019), "mapping is the creation of maps, a graphic symbolic representation of the significant features of a part of the surface of the Earth". At the same time, we will find out that there are also different meanings, because mapping can be found in:

- Cartography, mapping in general

○ Web mapping, mapping using data delivered by GISs

- Mapping (mathematics), a synonym for mathematical function or for morphism

- Gene mapping, the assignment of DNA fragments to chromosomes

- Brain mapping, set of techniques to study the brain

- Data mapping, data element mappings between two distinct data models

- Texture mapping, in computer graphics

- Mind mapping, the drawing of ideas and the relations among them

- Robotic mapping, creation and use of maps by robots

- Spiritual mapping, a practice of some religions

- Projection mapping, the projection of videos on the surface of objects with irregular shapes

\section{Definition of Mapping in Books}

If we look at the Glossary of the Mapping Sciences (1994) prepared by the Joint Committee of the American Society of Civil Engineering, American Society for Photogram-metry and Remote Sensing and American Congress on Surveying and Mapping, we will find the definition:

mapping - (1) The process of designing or making maps. The term as used by some cartographers to mean the making of planimetric or topographic maps only. It is used by some geographers and engineers to mean the gathering of geographical data of any sort. Neither usage is widespread and neither is particularly suitable. Both lead to difficulties. (2) A function or functional relationship. The term, used in this sense, is popular because of its pictorial associations, but is not suitable as a general synonym for function.
We can note from the quoted definition that mapping has different meanings depending on the person who is using it. Furthermore, according to the Glossary of Mapping Sciences, the given definitions are not suitable, although the title of the Glossary includes the term mapping. If we note that the Glossary contains more than 10,000 terms, then we can conclude that it was not easy to define mapping.

To say that the usage of the term mapping is not widespread is rather questionable in today's time. If, for example, we type mapping into the Google search engine, we will get about 241,000,000 addresses. Moreover, there are many books with the term mapping on their title pages (about 350 according to Google). They include:

Balakrishnan and Anderson (2012): Mapping the Nation (Mappings Series). Part of Verso's classic Mapping series that collects the most important writings on key topics in a changing world.

Bankoff et al. (2013): Mapping Vulnerability: Disasters, Development, and People. The book analyses 'vulnerability' as a concept central to the way we understand disasters and their magnitude and impact.

Carter (2013): Mapping the Mind. One of the clearest and best-illustrated attempts to explain the virtually inaccessible, the brain.

Crampton (2011): Mapping. A Critical Introduction to Cartography and GIS. An introduction to the critical issues surrounding mapping and Geographic Information Systems (GIS) across a wide range of disciplines for the non-specialist reader.

Cosgrove (1999): Mappings. The book explores what mapping used to mean and how its meanings have altered. How have maps and mapping served to order and represent physical, social and imaginative worlds? How has the practice of mapping shaped modern seeing and knowing? In what ways do contemporary changes in our experience of the world alter the meanings and practice of mapping, and vice versa?

Pile and Thrift (2005): Mapping the Subject: Geographies of Cultural Transformation. The book is an inter-disciplinary exploration of subjectivity, which focuses on the importance of space in acting, thinking, feeling individuals. 
Robinson and Petchenik (1976): The Nature of Maps: Essays toward Understanding Maps and Mapping. An introduction to a theory of cartography, attempting clear notions of the characteristics and processes by which a map acquires meaning from its maker and evokes meaning in its user.

Vaughan (2018): Mapping Society. The book traces the evolution of social cartography over the past two centuries. In this richly illustrated book, Laura Vaughan examines maps of ethnic or religious difference, poverty, and health inequalities, demonstrating how they not only serve as historical records of social enquiry, but also constitute inscriptions of social patterns that have been etched deeply on the surface of cities.

Wallis and Robinson (1987): Cartographical Innovations: An International Handbook of Mapping Terms to 1900.

Whitaker (2003): Mapping and Naming the Moon: A History of Lunar Cartography and Nomenclature. Beautiful lunar maps spanning four centuries of progress wonderfully illustrate the unfolding of our ability to map the Moon.

Žižek (2012): Mapping Ideology. Part of Verso's classic Mapping series that collects the most important writings on key topics in a changing world.

Obviously, term mapping is very modern nowadays. Although book titles include the term mapping, authors largely avoid defining it. This is also true for well-known cartographers such as H. Wallis and A. H. Robinson. In their International Handbook of Mapping Terms (1987), mapping is not defined.

Still, there are exceptions. For example, L. Vaughan begins the first chapter of his book Mapping Society with the words: "Despite its shifting meaning, mapping remains 'a way of representing the world', a visible image of, if not the world, then an aspect of that world." She refers to Cosgrove (1999), who wrote: "But the map as a tool of geographic science, and mapping as an active practice in the complex construction and communication of spatial knowledge, have been subjected to concentrated reflection, criticism and debate over the past quarter century, so that, like landscape, the map and mapping are now the subject of wide, interdisciplinary interest and use. ... Mapping remains a way of representing the world; the map remains a visible image of the (or at least $a$ ) world... As a graphic register of correspondence between two spaces, whose explicit outcome is a space of representation, mapping is a deceptively simple activity. ... Mapping is a process which involves both a 'complex architecture of signs' (graphic elements with internal forms and logics capable of theoretical disconnection from any geographical reference) and a 'visual architecture' through which the worlds they construct are selected, translated, organized and shaped."

\section{Everybody Knows What Mapping is?}

Apart from books, there are other sources one might think contain the definition of mapping. Unfortunately, this is not always true. I will only mention two examples. For instance, the famous cartographer Christopher Board (1982) wrote a paper Maps and mapping. Unfortunately his paper does not include definitions of map and mapping.

Another example is the web-site of ICSM (The Intergovernmental Committee on Surveying and Mapping, from 2002 a Standing Committee of ANZLIC - the Australian and New Zealand Spatial Information Council). There is a site titled Fundamentals of Mapping (ICSM 2019) designed to provide a comprehensive general overview of maps, mapping, cartography and map production. It is not intended to be a definitive reference, but rather to supply a consolidated summary of mapping concepts, principles and practice. A visitor of this site will have to imagine what the meaning of mapping is, because the definition is missing.

\section{Mapping in Map Projections}

Mapping is one of the basic terms in mathematics. To recall, two non-empty sets, $X$ and $Y$, are given. By mapping we understand the rule that each element $x$ from $X$ joins element $y$ from $Y$. So, we write $f: X \rightarrow Y$. Element $y$ is the image of $x$, so we write $y=f(x)$. Set $X$ is the domain of mapping $f$, while set $Y$ is codomain.

Map projections are, consciously or unconsciously, the foundation of each map. Assuming that the concept of mapping is known from mathematics (it should be, because it has always been around in education), we can define a map projection as the mapping of a curved surface, especially a sphere or ellipsoid, into a plane. So why not use the term mapping, when it is so neatly linked to the word map?

\section{A Questionnaire}

The $7^{\text {th }}$ International Conference on Cartography and GIS was held in Sozopol, Bulgaria, in 2018. A meeting of the ICA Commission on Map Projections was organized as a part of this conference. As a Chair of the Commission, I asked the participants of the meeting to finish in written form the sentence "Mapping is ...". Their answers, including some more obtained later, were:

Serenity, Accuracy, Meanings, Sharing, K.N.O.W. L.E.D.G.E, an invitation to ever ask and never believe, From 4 to 177 !

- Selecting, processing and transforming geospatial data to represent real or synthetic phenomena in a form useful to human and machine interpretation and application to solve problems.

- Magic.

- Creating a window to the world.

- A beautiful activity $\odot$ representing reality!

- To transfer the spatial or spatio-temporal information collected during the survey, spatial and/or spatiotemporal analysis into the mathematic system of coordinates to create the map.

- Matching elements from one model to the appropriate equivalents (elements of the other model that includes 
also relations between particular elements or groups of elements).

- A useful art.

- Process of representing geo-information received from imagery, photogrammetrical-geodetical measurements, etc. on different platforms and devices in vector or raster form according chosen coordinate system and map projection.

- A scientific process of depicting the earth surface and objects over it from knowledgeable people with classical and modern visualization methods in 2D/3D space with some distortion.

- The effort to provide context to our real-world phenomena on 2D means and to support our understanding of world, entrepreneurship, and cooperation between various fields, people and countries.

- Mapping is the application of a surjective function from a subset of a (virtual) reality [mini-world] as domain to a generalized (analogue or digital) representation [spatial model] as co-domain.

- Actually, in a very general sense I would define it as an activity to record/document/capture entities and their relations. The recording would be usually WYSIWYG, thus graphically and visually perceivable, but could be done eventually differently as well. The entities can be all kind of objects, primarily spatial objects. The relations are distances, positions, topologies, thus spatial relations.

- Mapping is the process of creating a map, which is a device that uses graphical language to communicate reality (or a theoretical concept) beyond our immediate comprehension by preserving spatial arrangements and by simplifying detail. I suppose I draw a distinction between mapmaking and mapping in that the former implies the creation of an artefact while the latter is a process that may be undertaken without the desire to make an object. Mapping and cartography to me are analogous to building and architecture; the former being the process of constructing something the latter representing the whole from conception and design to creating an object.

As we can see, there are 14 different definitions of mapping. All of these definitions were given by professional cartographers.

\section{Conclusion}

As a result, it is not difficult to conclude that the meaning of the term mapping in cartographic community is rather loose. It follows, that if somebody uses this term, they need to define or clarify its meaning. Otherwise, certain misunderstanding can be expected.

To clarify the situation, I would propose the definition of mapping in cartography or cartographic mapping as a process that associates a set of spatially related data with another set called a representation or model or map, while preserving spatial arrangements and by simplifying detail.
If we accept this definition of mapping, then we can easily derive the definitions of map and cartography.

A map is a result of cartographic mapping.

Cartography is the art, science and technology of mapping and using maps.

\section{Acknowledgements}

The author wishes to thank the anonymous reviewer for his/her valuable comment, as well as to colleagues who participated in the survey on the definition of mapping.

\section{References}

Balakrishnan, G., Anderson, B. (2012).Mapping the Nation (Mappings Series), Verso Books.

Bankoff, G., Frerks, G., Hilhorst, D. (2013).Mapping Vulnerability: Disasters, Development, and People, Earthscan.

Board, Ch. (1982). Maps and mapping, Progress in Human Geography, Vol. 6, No. 1, 106-113

Carter, R. (2013).Mapping the Mind, Hachette UK

Crampton, J. W. (2011). Mapping, A Critical Introduction to Cartography and GIS, Wiley-Blackwell Cosgrove, D. (1999). Mappings, Reaktion Books, 1999

Glossary of the Mapping Sciences (1994). American Society of Civil Engineering, American Society for Photogrammetry and Remote Sensing and American Congress on Surveying and Mapping

ICSM (2019): Fundamentals of mapping, https://www. icsm.gov.au/education/fundamentals-mapping, (accessed 3 Jan 2019)

Pile, S., Thrift, N. (2005).Mapping the Subject: Geographies of Cultural Transformation, Routledge

Robinson, A. H., Petchenik B. B. (1976): The Nature of Maps: Essays toward Understanding Maps and Mapping,University of Chicago Press

Vaughan, L. (2018): Mapping Society, The Spatial Dimension of Social Cartography, UCL Press

Wallis, H., Robinson A. H. (1987): Cartographical Innovations: An International Handbook of Mapping Terms to 1900, Map Collector Publications Ltd.

Whitaker, E. A. (2003): Mapping and Naming the Moon: A History of Lunar Cartography and Nomenclature. Cambridge University Press

Wikipedia (2019): Mapping (accessed 3 Jan 2019)

Žižek, S. (2012): Mapping Ideology, Verso Books 